
anabolic androgenic steroids in sport. Journal of Education, Health and Sport. 2020;10(9):349-356. eISSN 2391-8306. DOI http://dx.doi.org/10.12775/JEHS.2020.10.09.040

https://apcz.umk.pl/czasopisma/index.php/JEHS/article/view/JEHS.2020.10.09.040

https://zenodo.org/record/4030051

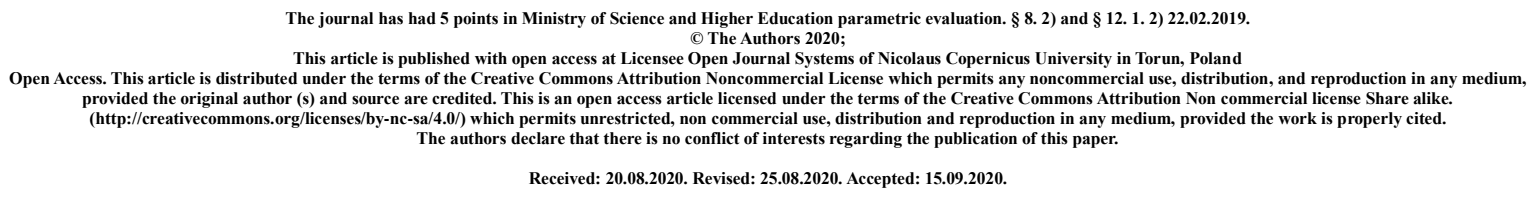

Hepatotoxicity of anabolic androgenic steroids in sport

\author{
Michał Sekuła, Blanka Świerczyńska, Krzysztof Smoluchowski, Adrian Undziakiewicz, \\ Halina Piecewicz-Szczęsna
}

\author{
Student Research Circle at the Department of Epidemiology and Clinical Research \\ Methodology, Medical University of Lublin
}

\begin{abstract}
Introduction and purpose: Anabolic-androgenic steroids (AAS) abuse is a common practice among professional as well as recreational athletes. The prevalence of this phenomenon seems to be continuously escalating, too. Even though AAS abuse may provide a desirable haematopoietic and musculotrophic effect, it causes a wide variety of local and systemic side effects. This review aims to demonstrate the AAS induced hepatotoxicity, it is based on the medical literature from the last 10 years.

Brief description of the state of knowledge: Orally administered 17- $\alpha$-alkylated steroids are considered to be the most hepatotoxic. They increase the number of reactive oxygen species (ROS) which directly injure the liver tissue. It may be manifested as an elevation of liver enzymes, peliosis hepatis or distinctive acute cholestasis. Furthermore, ASS abuse for a prolonged period of time may induce the growth of hepatocellular adenoma and hepatocellular cancer. Moreover, AAS intake can lead to liver enlargement, development of toxicant-associated fatty liver disease (TAFLD) and increase the risk of $\mathrm{HCV}$ and $\mathrm{HBV}$ infection.
\end{abstract}


Conclusions: The collaboration of scientists, doctors, sports associations and governments is substantial. Introduction of innovative AAS detection methods and enforcement of stricter anti-doping law may contribute to prevention of health consequences resulting from AAS abuse among athletes.

Key words: hepatotoxicity, anabolic androgenic steroids, liver injury, sport

\section{Introduction and purpose:}

Anabolic-androgenic steroids (AAS) abuse is a common practice among professional as well as recreational athletes. The prevalence of this phenomenon seems to be continuously escalating, too[43]. The World Anti-Doping Agency (WADA) reports that the number of samples collected from athletes is annually increasing. Bodybuilding, athletics, cycling and weightlifting are characterized by the highest use of AAS and other doping substances [1-4]. AAS are used as doping substances in significantly higher doses than the clinical ones [5,9,11,13], sometimes without maintaining a proper cyclic pattern of intake [39]. Even though AAS abuse may provide a desirable haematopoietic and musculotrophic effect [5], it causes significant damage to a reproductive system[6], cardiovascular system, liver, kidneys, muscles and psyche [5,12]. Furthermore, frequent AAS intramuscular injections can lead to local adverse effects such as fibrodysplasia ossificans [7]. It seems that some of the athletes are not aware of health consequences attributed to inappropriate AAS intake [19]. They are also reluctant to admit to AAS abuse which may cause a serious diagnostic challenge $[5,11]$. It turns out that some of the dietary supplements and herbal products contain AAS without an indication on the etiquettes, which leads to an unintentional intake [8-11, 13]. Moreover, multiple athletes take AAS in different forms together with other substances to increase effectiveness or diminish the side effects, which leads to polypragmasie [5,8,12,39-41,44]. This review aims to demonstrate the AAS induced hepatotoxicity, it is based on the medical literature from the last 10 years.

\section{State of knowledge:}

\subsection{Hepatotoxity of steroids}

AAS are synthetic derivatives of testosterone. Testosterone is a natural steroid hormone that can be found in a male body and in a female body but in the lower quantity. In order to reduce the undesirable androgenic effect and increase the bioavailability of the oral AAS, new synthetic derivatives of testosterone have been investigated since the discovery of testosterone in 1935 [14]. There are two main groups of testosterone derivatives:

a) modificated by esterification of the 17- $\beta$-hydroxyl group (testosterone cypionate, enanthate and propionate) - characterized by a higher potency and a longer duration of action $[8,13]$, they have to be administered intravenously [15];

b) modificated by 17- $\alpha$-alkylation - they can be administered orally, but due to decreased hepatic metabolism they seem to be more hepatotoxic $[8,13,15]$. This group includes: methyltestosterone, methandrostenolone, oxymetholone, danazol, epistane, fluoxymesterone, stanozolol, norethandrolone and oxandrolone [8]. 
C-17- $\alpha$ alkylated androgenic steroids are responsible for most of the hepatotoxic adverse effects: elevated liver enzymes, cholestatic jaundice, peliosis hepatis, hepatic adenoma and hepatocellular carcinoma [11]. 17- $\beta$ - hydroxy esters are undoubtedly less hepatotoxic, but a prolonged use can induce nodular transformation or a tumour growth $[8,13]$. Nortestosterone (nandrolone) consists of another modification - 19-demethylation [8].

Unfortunately, C-17 $\alpha$-alkylated steroids abuse is the most common among athletes trying to increase their muscle mass and performance [13]. Researchers have noticed a major escalation of AAS induced hepatic diseases in the last two decades[9]. The mechanism of hepatotoxicity is still unclear. Nevertheless, it is most likely an effect of an oxidative stress inside the hepatic cells $[17,18]$. Carnitine palmitoyltransferase I (CPTI), a rate-limiting enzyme of mitochondrial fatty acids $\beta$-oxidation, may be responsible for enlarging the number of reactive oxygen species (ROS) [14]. By activating androgen receptors (ARs), AAS can boost the CPT1 activity. It leads to an increased mitochondrial fatty acids $\beta$-oxidation and production of ROS [14].

\subsection{Types of liver injury}

\section{Anabolic steroids and hepatic volume}

AAS, likewise testosterone, may cause dose-related increase in the hepatic volume. It complies with the research performed on the animals and the fact that male liver is statistically larger than a female one [23].

\section{Elevated liver enzymes}

The injury of hepatic cells causes an elevation of enzymes such as: aspartate aminotransferase (AST), alanine aminotransferase (ALT), gamma glutamyl transpeptidase (GGT), alkaline phosphatase (AP) and lactate dehydrogenase (LDH) [8,13,33]. Steroid hormones abuse usually causes an increased hepatic hormones quantities in blood plasma. Generally, AST and ALT levels do not exceed the 2-3 times the upper limit normal, they are symptompless and self limiting [13,22,32,33]. Elevated liver enzymes are typical for danazol and oxymetholone abuse [13]. The levels of aminotransferases normalise after a few weeks of steroid withdrawal $[20,21,32]$. Intensive training may cause a creatine kinase (CK) and AST elevation, however it does not increase the GGTP level [8]. Even among people who do not abuse ASS, an increased AST and ALP values may be a rhabdomyolysis symptom, especially when CK is increased, too [12]. Assessment of the liver function and ASS induced hepatotoxicity based merely on the liver enzymes levels can be misleading and cause an overestimation of a problem $[8,12]$.

\section{Acute cholestatic syndrome}

Acute cholestatic syndrome is a very common injury caused by AAS abuse [13,32,43]. It should be considered even when a patient does not admit to take AAS or reports administration of herbal products or dietary supplements that do not have AAS listed on the label [24,25]. The incidence of acute cholestasis is equal to about $1 \%$ among patients abusing AAS such as: methyltestosterone, danazol, stanozolol or oxymetholone and it seems to be dose-related [11,13]. The injury of the liver usually occurs after 1-4 months of AAS use, but it may be delayed up to 6-24 months [24,26]. 
Animal research indicates that this injury, caused by $\mathrm{C}-17 \alpha$ alkylated androgenic steroids, may be an effect of disrupted intrahepatic microfilaments and diminished number of bile salt transporter proteins [9,11]. Liver cholestasis is rare in patients taking unmodified testosterone orally or subcutaneously [13,26]. Nonetheless, one case of such a patient has been published [31]. The beginning of the cholestatic liver injury manifestation is deceptive, patients suffer from nausea, fatigue and itching followed by dark urine and jaundice [8,26,32]. Hyperbilirubinemia is accompanied by an insignificant elevation of aminotransferases. AP level may be normal or just slightly increased at first, but it gradually escalates later on. GGTP usually stays unaffected [9,32]. Pathological examination of biopsy material often reveals a cholestasis with slight inflammation and hepatic cells necrosis [9,24]. In most cases acute cholestasis is temporary and declines after AAS withdrawal. However, occasionally it may prolong or turn into more severe form [9,26,32]. Sporadically, severe cholestasis is followed by an acute renal injury that may even demand renal replacement therapy [28, 29,43]. Cholestyramine, ursodiol and antihistamine drugs (which alleviate the itching) are used in the treatment of cholestasis but they do not seem to lessen the liver damage or accelerate the regeneration process [26, 27]. Even though steroid hormones should not be applied in the acute cholestasis treatment, one case study of a patient successfully treated with modest doses of steroid hormones has been published [30].

\section{Peliosis Hepatis}

Long-term AAS abuse may induce an uncommon vascular condition called peliosis hepatis. It was discovered in 1952, but the pathological mechanism remains unknown $[11,15]$. It is characterised by enlarged hepatic sinuses and cysts filled with blood, that are either singular or multiple and disseminated throughout the liver [8,11,13]. It is most often accompanied by sinusoidal dilatation and loss of the regular endothelial barrier $[8,13]$. The liver may be enlarged, deep red in color and fragile $[8,11,13]$. Peliosis hepatis is usually found in patients with severe and devastating diseases such as cancer. However it may also occur in patients undergoing a prolonged steroid drugs treatment for aplastic anemia, people with hypogonadism as well as athletes abusing ASS to enhance their sports performance $[11,13]$. Peliosis hepatis is usually symptomless, sometimes patients may experience discomfort localised in the right upper quadrant of stomach with a palpable enlargement of liver $[8,13]$. Most frequently the liver enzymes are not elevated [13]. Sporadically patients may suffer from acute stomach pain and hypovolemic shock as a symptom of liver laceration with formation hemoperitoneum, a potentially lethal condition [34]. Treatment of peliosis hepatis is only symptomatic. The regression of cysts is possible after AAS withdrawal [8]. Peliosis may influence other organs, such as a spleen, too $[13,15]$.

\section{Hepatic Tumors}

AAS intake may lead to rare but dangerous adverse effects, such as hepatocellular adenomas (HCA) and hepatocellular cancer (HCC) (firstly reported in the 1950s) [5, 11]. These tumours are usually developed by patients using AAS for a prolonged period of time because of medical conditions such as aplastic anemia, hypogonadism or gender identity disorder $[8,13,38]$ as well as athletes abusing AAS as a doping substance $[8,13]$. 
It usually takes about a few years of AAS abuse for a tumour to develop, nonetheless more rapid growth has been reported, too $[8,11,13]$. Patients suffering from other risk factors such as Fancony's anemia or chronic B/C hepatitis are more susceptible to tumour formation [11,13]. HCA and HCC can be induced by both oral and parenteral AAS administration [11]. However, it seems that 17- $\alpha$ - alkylated steroids are the most tumorigenic [8]. The risk of cancer development is possibly linked to the cumulative dose of a substance and its potency [15]. HCCs, HCAs and hepatocellular adenomas with malignant transformation zones $[8,13]$ may be difficult to distinguish during the pathological examination $[38,40]$. The cases of cholangiocarcinoma and angiosarcoma among AAS users have been described in the literature, too $[8,13]$. HCAs are benign liver tumours, commonly induced by oral contraceptives used by young women [38], sometimes by AAS abuse, as well $[8,15]$. $\beta$ catenin mutation, tumour diameter $>5 \mathrm{~cm}$ and male sex are risk factors [8] for malignant transformation of HCAs. It is uncommon [11] and occurs in about $4.5 \%$ to $9 \%$ of all cases. $4.2 \%$ of HCA will have actual foci of HCC [8]. It seems that AAS usually induces formation of multiple adenomas rather than one [37-40]. HCA may rupture which can lead to lifethreatening hemorrhagic shock and hemoperitoneum, demanding an urgent surgical intervention $[8,11,37,39]$. That is why a biopsy of HCAs should be performed under extreme caution or should not be done at all [37]. HCC is hardly ever induced by AAS abuse [40]. It appears that it is linked to a better prognosis than HCC caused by hepatitis C, B or cirrhosis hepatis $[8,13,40,41]$. The symptoms of liver tumours are: sensation of pain and palpable masses in the right upper quadrant of the stomach, radiological evidence of a tumour $[8,13,37,41]$, occasionally followed by cholestasis [37,42]. As long as there are neither additional liver injuries nor tumour related emergencies, the hepatic enzymes and $\alpha$ fetoprotein levels are not elevated $[8,13,37,41]$. While establishing a treatment strategy it is important to keep in mind that HCAs (especially when detected early) may undergo a spontaneous regression [5,8,11,37,39]. Progression of HCAs as well as their recurrence have been reported in patients who did not withdraw AAS [8].

\section{Other forms of liver injury}

Toxicant-associated fatty liver disease (TAFLD) may be caused by AAS abuse $[5,35,36]$. The mechanism is still unrecognized, however the direct toxic influence of AAS is suspected [35,36]. Moreover, the intravenous injections of ASS may increase the risk of HCV and HBV infection [8].

\section{Conclusions}

AAS abuse without neither medical indications nor a doctor consultation constitutes a major problem. It affects mostly young men who try to enhance their sport performance and improve their appearance. The magnitude of this phenomenon and the number of related side effects (e.g. hepatotoxicity) seems to be increasing in the last decades. Reckless use of AAS can cause a temporary liver dysfunction, cholestasis, peliosis hepatis or even liver tumours growth. It may also seriously damage the reproductive system, cardiovascular system, kidneys, muscles, skin and psyche. In some cases it may lead to a patient's death. Doctors, especially ones specialized in sports medicine, should be aware of the AAS abuse problem and be familiar with the list of prohibited substances. 
Moreover, they should know the symptoms of AAS abuse and correlated side effects . Introduction of innovative diagnostic procedures as well as the extensive efforts of both national and international anti-doping agencies are required to enhance the methods of antidoping control. The problem of AAS abuse among recreational athletes should be addressed, too. Finally, sport associations together with individual countries' governments should aspire to tighten up the regulations. They should also consistently punish the deliberate abuse of AAS and other doping substances in order to diminish the problem and related health consequences.

\section{References:}

[1] 2017 Anti-Doping Rule Violations (ADRVs) Report https://www.wadaama.org/sites/default/files/resources/files/2017_adrv_report.pdf

[2] 2016 Anti-Doping Rule Violations (ADRVs) Report https://www.wadaama.org/sites/default/files/resources/files/2016 adrvs_report_web_release april_2018_0.pdf

[3] 2015 Anti-Doping Rule Violations (ADRVs) Report https://www.wadaama.org/sites/default/files/resources/files/2015_adrvs_report_web_release 0.pdf

[4] 2014 Anti-Doping Rule Violations (ADRVs) Report https://www.wadaama.org/sites/default/files/resources/files/wada-2014-adrv-report-en_0.pdf

[5] Eberhard Nieschlag, Elena Vorona, Doping with anabolic androgenic steroids (AAS): Adverse effects on non-reproductive organs and functions, Rev Endocr Metab Disord. 2015 Sep;16(3):199-211

[6] Eberhard Nieschlag, Elena Vorona, MECHANISMS IN ENDOCRINOLOGY: Medical consequences of doping with anabolic androgenic steroids: effects on reproductive functions in European Journal of Endocrinology, R47-R58, Volume 173: Issue 2

[7] Mark M Schultzel, Michael H Johnson, Howard G Rosenthal, Bilateral deltoid myositis ossificans in a weightlifter using anabolic steroids, Orthopedics. 2014 Sep;37(9):e844-7

[8] Mark W Niedfeldt, Anabolic Steroid Effect on the Liver, Curr Sports Med Rep. 2018 Mar;17(3):97-102.

[9] Ammar Hassan, Robert J Fontana, Liver Injury Associated with Sporting Activities, Semin Liver Di. 2018 Nov;38(4):357-365.

[10] Inmaculada Medina-Caliz, Miren Garcia-Cortes, Andres Gonzalez-Jimenez I wsp., Herbal and Dietary Supplement-Induced Liver Injuries in the Spanish DILI Registry, Clin Gastroenterol Hepatol. 2018 Sep;16(9):1495-1502.

[11] R Solimini, M C Rotolo, L Mastrobattista, C Mortali, A Minutillo, S Pichini, R Pacifici, I Palmi, Hepatotoxicity associated with illicit use of anabolic androgenic steroids in doping, Eur Rev Med Pharmacol Sci. 2017 Mar;21(1 Suppl):7-16.

[12] Harrison G. Pope, Jr., Ruth I. Wood, Alan Rogol, Fred Nyberg, Larry Bowers, and Shalender Bhasin, Adverse Health Consequences of Performance-Enhancing Drugs: An Endocrine Society Scientific Statement, Endocr Rev. 2014 Jun; 35(3): 341-375.

[13] Androgenic Steroids, National Institute of Diabetes and Digestive and Kidney Diseases; 2012-.2020 May 30.

[14] Peter Bond, William Llewellyn, Peter Van Mol, Anabolic androgenic steroid-induced hepatotoxicity, Med Hypotheses. 2016 Aug;93:150-3.

[15] M Neri, S Bello, A Bonsignore, S Cantatore, I Riezzo, E Turillazzi, V Fineschi, Anabolic androgenic steroids abuse and liver toxicity, Mini Rev Med Chem. 2011 May;11(5):430-7.

[16] Cerretani, D., Neri, M., Cantatore, S., Ciallella, C., Riezzo, I., Turillazzi, E., Fineschi, V., Looking for organ damages due to Anabolic-androgenic Steroids (AAS): Is oxidative stress the culprit?, Mini-Reviews in Organic Chemistry Volume 10, Issue 4, 2013, Pages 393-399

[17] Mostafa El-Moghazy, Ehab Tousson, Changes in the hepatic and renal structure and function after a growth promoter boldenone injection in rabbits, Animal Biology, Volume 62: Issue 2, 171-180 
[18] Stephan P. Frankenfeld, Leonardo P. Oliveira, Victor H. Ortenzi, Igor CC. Rego-Monteiro, Elen A. Chaves, Andrea C. Ferreira, Alvaro C. Leitão, Denise P. Carvalho and Rodrigo S. Fortunato, The Anabolic Androgenic Steroid Nandrolone Decanoate Disrupts Redox Homeostasis in Liver, Heart and Kidney of Male Wistar Rats, PLoS One. 2014; 9(9): e102699.

[19] Amer A Almaiman, Sulaiman H Almaiman, Elsayed I Elagamy, Osama Al Wutayd, Mohammed Almarzuqi, Rayyan Alzunaidi, Sami Alhatlani, Eltayeb E Eid, Side effects of anabolic steroids used by athletes at Unaizah Gyms, Saudi Arabia: a pilot study, J Sports Med Phys Fitness. 2019 Mar;59(3):489-495.

[20] Polly Carson, Christine J Hong, Marta Otero-Vinas, Emily Frances Arsenault, Vincent Falanga, Liver enzymes and lipid levels in patients with lipodermatosclerosis and venous ulcers treated with a prototypic anabolic steroid (stanozolol): a prospective, randomized, double-blinded, placebo-controlled trial, Int J Low

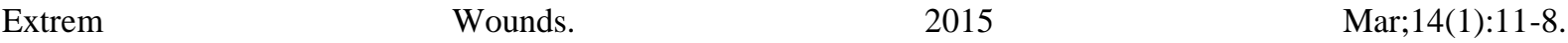

[21] Shehzad Basaria, Androgen abuse in athletes: detection and consequences, J Clin Endocrinol Metab. 2010 Apr;95(4):1533-43.

[22] Roseman DA, Hwang SJ, Oyama-Manabe N, Chuang ML, O’Donnell CJ, Manning WJ, Fox CS. Clinical associations of total kidney volume: the Framingham Heart Study. Nephrol Dial Transplant 2016.

[23] T Gagliano-Jucá, E R Tang, S Bhasin, K M Pencina, S Anderson, H Jara, Z Li, K Melamud, S L Coleman, A Aakil, R R Almeida, G Huang, T G Travison, T W Storer, S Basaria, Effects of testosterone administration (and its 5-alpha-reduction) on parenchymal organ volumes in healthy young men: findings from a dose-response trial, Andrology. 2017 Sep;5(5):889-897.

[24] Ahmed M Elsharkawy, Stuart McPherson, Steven Masson, Alastair D Burt, Robert T Dawson, Mark Hudson, Cholestasis secondary to anabolic steroid use in young men, BMJ. 2012 Feb 2;344:e468

[25] Michel I Kafrouni, Robert A Anders, Sumita Verma, Hepatotoxicity associated with dietary supplements containing anabolic steroids, Clin Gastroenterol Hepatol. 2007 Jul;5(7):809-12.

[26] Brett M Hymel, David W Victor, Luis Alvarez, Nathan J Shores, Luis A Balart, Mastabol induced acute cholestasis: A case report, World J Hepatol. 2013 Mar 27;5(3):133-6. [27] Victor J Navarro, Ikhlas Khan, Einar Björnsson, Leonard B Seeff, Jose Serrano, Jay H Hoofnagle, Liver injury from herbal and dietary supplements, Hepatology. 2017 Jan;65(1):363-373.

[28] Ahmed M Alkhunaizi, Mohamed A ElTigani, Rola S Rabah, Samih H Nasr, Acute bile nephropathy secondary to anabolic steroids, Clin Nephrol. 2016 Feb;85(2):121-6.

[29] Mónica Milla Castellanos, Eduardo Gutiérrez Martínez, Ángel Sevillano Prieto, Paola Rodríguez Ramos, Manuel Praga Terente, Bile cast nephropathy associated with severe liver dysfunction caused by anabolic steroids, Nefrologia. Mar-Apr 2018;38(2):221-223

[30] Piotr M. Stępień, Katarzyna Reczko, Anna Wieczorek, Dorota Zarębska-Michaluk, Paweł Pabjan, Teodora Król and Wiesław Kryczka, Severe intrahepatic cholestasis and liver failure after stanozolol usage - case report and review of the literature, Clin Exp Hepatol. 2015 May; 1(1): 30-33.

[31] Marije N Boks, Anton T Tiebosch, Laurens A van der Waaij, A jaundiced bodybuilder Cholestatic hepatitis as side effect of injectable anabolic-androgenic steroids, J Sports Sci. 2017 Nov;35(22):2262-2264.

[32] Charles D Magee, Stephen Witte, Ryan M Kwok, Patricia A Deuster, Mission Compromised? DrugInduced Liver Injury From Prohormone Supplements Containing Anabolic-Androgenic Steroids in Two Deployed U.S. Service Members, Mil Med. 2016 Sep;181(9):e1169-71.

[33] Amer A Almaiman, Sulaiman H Almaiman, Elsayed I Elagamy, Osama Al Wutayd, Mohammed Almarzuqi, Rayyan Alzunaidi, Sami Alhatlani, Eltayeb E Eid, Side effects of anabolic steroids used by athletes at Unaizah Gyms, Saudi Arabia: a pilot study, J Sports Med Phys Fitness. 2019 Mar;59(3):489-495.

[34] Sun-Keun Choi, Jun-Shuo Jin, Soon-Gu Cho, Suk-Jin Choi, Chul-Soo Kim, Yun-Mee Choe, and KeonYoung Lee, Spontaneous liver rupture in a patient with peliosis hepatis: A case report, World J Gastroenterol. 2009 Nov 21; 15(43): 5493-5497.

[35] Paulo Adriano Schwingel, Helma P Cotrim, Bernardo Rios Salles, Carlos Eduardo Almeida, Crimério Ribeiro dos Santos Jr, Bruno Nachef, Antonio Ricardo Andrade, Cláudio C Zoppi, Anabolic-androgenic steroids: a possible new risk factor of toxicant-associated fatty liver disease, Liver Int. 2011 Mar;31(3):348-53

[36] Paulo Adriano Schwingel, Helma Pinchemel Cotrim, Crimério Ribeiro dos Santos Jr, Adriano Oliveira dos Santos, Antônio Ricardo Cardia Ferraz de Andrade, Marcos Vinicius Vilas Boas Carruego, Cláudio Cesar Zoppi, Recreational Anabolic-Androgenic Steroid Use Associated With Liver Injuries Among Brazilian Young Men, Subst Use Misuse. 2015;50(11):1490-8. 
[37] Lixia Wang, Cong Wang, Wei Li, Fanyang Meng, Yuying Li, Hongqiong Fan, Yanhua Zhou, Gnana Bharathi, Sujun Gao, Yan Yang, Multiple hepatocellular adenomas associated with long-term administration of androgenic steroids for aplastic anemia: A case report and literature review, Medicine (Baltimore). $2020 \mathrm{Jul}$ 10;99(28):e20829.

[38] Keizo Kato, Hiroshi Abe, Noriko Hanawa, Junya Fukuzawa, Ryota Matsuo, Takeshi Yonezawa, Sadahiro Itoh, Yoshiyuki Sato, Makiko Ika, Shohei Shimizu, Shinji Endo, Hiroshi Hano, Asami Izu, Masahiko Sugitani, Akihito Tsubota, Hepatocellular adenoma in a woman who was undergoing testosterone treatment for gender identity disorder, Clin J Gastroenterol. 2018 Oct;11(5):401-410.

[39] D L Smit, J H Nuijens, W de Ronde, Spontaneous haemorrhage of hepatic adenoma in a patient addicted to anabolic steroids, Neth J Med. 2019 Sep;77(7):261-263.

[40] Philipp Solbach, Andrej Potthoff, Hans-Jürgen Raatschen, Bisharah Soudah, Ulrich Lehmann, Andrea Schneider, Michael J Gebel, Michael P Manns, Arndt Vogel, Testosterone-receptor positive hepatocellular carcinoma in a 29-year old bodybuilder with a history of anabolic androgenic steroid abuse: a case report, BMC Gastroenterol. 2015 May 20;15:60.

[41] Aline Hardt, Dirk Stippel, Margarete Odenthal, Arnulf H Hölscher, Hans-Peter Dienes, Uta Drebber, Development of hepatocellular carcinoma associated with anabolic androgenic steroid abuse in a young bodybuilder: a case report, Case Rep Pathol. 2012;2012:195607

[42] Sounak Gupta, Bita V Naini, Richard Munoz, Rondell P Graham, Benjamin R Kipp, Michael S Torbenson, Taofic Mounajjed, Hepatocellular Neoplasms Arising in Association With Androgen Use, Am J Surg Pathol. $2016 \mathrm{Apr} ; 40(4): 454-61$

[43] M Robles-Diaz, A Gonzalez-Jimenez, I Medina-Caliz, C Stephens, M García-Cortes, B García-Muñoz, A Ortega-Alonso, E Blanco-Reina, R Gonzalez-Grande, M Jimenez-Perez, P Rendón, J M Navarro, P Gines, M Prieto, M Garcia-Eliz, F Bessone, J R Brahm, R Paraná, M I Lucena, R J Andrade, Spanish DILI Registry; SLatinDILI Network, Distinct phenotype of hepatotoxicity associated with illicit use of anabolic androgenic steroids, Aliment Pharmacol Ther. 2015 Jan;41(1):116-25.

[44] Mario Thevis, Tiia Kuuranne Hans Geyer, Annual banned-substance review - Analytical approaches in human sports drug testing, https://doi.org/10.1002/dta.2735 